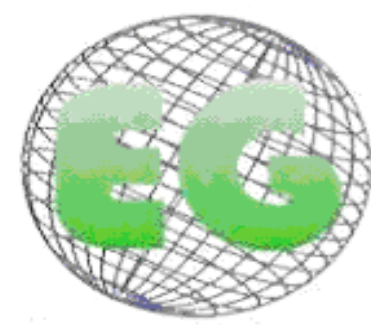

ISSN 1695-6141 N'25

\title{
Percepción de los estudiantes de enfermería acerca de su protección ante patologías inmunoprevisibles
}

Percepção dos acadêmicos de enfermagem acerca da sua proteção à patologias imunopreviníveis

\author{
*Wojciechowski de Carvalho, M do C., "Zepka Baumgarten, L., " ${ }^{* * *}$ Riegert Borba, \\ M., ${ }^{* * * *}$ Silva Da Costa, CF.
}

\author{
*Enfermera. ${ }^{* *}$ Msc. en Enfermería. Docente de la Escuela de Enfermería de la Universidad Federal de Rio \\ Grande FURG. Brasil.
}

Palabras clave: estudiantes de enfermería, vacunación; enfermería

Palavras-chave: estudiantes de enfermagem; vacinação; enfermagem

Keywords: nursing students; vaccination; nursing

\section{RESUMEN}

El objetivo de este estudio fue identificar el conocimiento de los estudiantes del curso de enfermería de una universidad federal del sur del País acerca de su protección a patologías inmunoprevisibles. Se sometieron a un estudio cualitativo de tipo exploratorio 35 estudiantes de enfermería a los cuales se aplicó un cuestionario con preguntas abiertas. Las respuestas originaron las siguientes categorías de análisis: importancia de la inmunización; actividades de riesgo; acceso a las informaciones sobre inmunización; vacunas indicadas a los trabajadores de salud; sugerencias para contextualizar la necesidad de inmunización. Los resultados revelaron que a pesar de que los estudiantes consideraron importante la inmunización como um modo de protección, el abordaje del tema en su formación todavía es muy pequeño. Esto se debe a las barreras relacionadas, como la falta de una reflexión acerca del asunto en la institución que oriente y respalde la acción profesional. Fue posible identificar también que los estudiantes reconocen las inmunizaciones como modos de protección y enfatizan la importancia de la prevención, sin embargo, muchos no tienen su calendario vacunal completado. Hacer obligatorio el comprobante de vacunación actualizado para todos los que tienen contacto en trabajos de prácticas, dará una mayor visibilidad al problema y retorno de la inversión de la institución. Así, se hace necesario un enfoque más específico de protección y prevención de los trabajadores durante la formación académica.

\section{RESUMO}

O objetivo deste estudo foi identificar o conhecimento específico dos acadêmicos do curso de enfermagem de uma universidade federal do sul do País, acerca da sua proteção a patologias imunopreveníveis. Foram submetidos a um estudo qualitativo do tipo exploratório 35 acadêmicos de enfermagem, aos quais foi aplicado um questionário com perguntas abertas. As respostas originaram categorias de análise, assim apresentadas: importância da imunização; atividades de risco; acesso às informações sobre imunização; vacinas indicadas aos trabalhadores da saúde; sugestões para contextualizar a necessidade de imunização. Os resultados revelaram que apesar dos acadêmicos considerarem importante a imunização como um modo de proteção, a 
abordagem do tema em sua formação ainda é muito pequena. Isso decorre dos entraves relacionados, como a falta de uma reflexão acerca do assunto, na instituição que oriente e respalde a ação profissional. Foi possível identificar também que os estudantes reconhecem as imunizações como modos de proteção e enfatizam a importância da prevenção, no entanto, muitos não possuem seu calendário vacinal completado. Tornar obrigatório o comprovante de vacinação atualizado para todos que têm contato com campos de prática, resultará numa maior visibilidade ao problema e retorno do investimento da instituição. Assim, faz-se necessário um enfoque mais específico de proteção e prevenção dos trabalhadores durante a formação acadêmica.

\section{ABSTRACT}

The aim of this study was to measure the knowledge of protection against vaccine preventable pathologies held by students on a nursing course at a federal university in the south of the country. The qualitative, exploratory study comprised 35 students of nursing who were given an open question questionnaire. The answers gave rise to the following analysis categorie: the importance of vaccination; risk activities; access to information about vaccination; recommended vaccinations for health workers; suggestons to contextualise the need for vaccinations. The results revealed that while the students saw vaccination as important for their protection, the issue is little addressed in the course of their training. This is due to certain barriers such as alack of consideration about the issue within the institution that directs and supports professional action. The study also found that students recognised vaccinations as a means of protection and stressed theimportance of prevention. However, many of them were not up to date with their own vaccination calendars. Making this obligatory for all in the fields of practice would serve to enhance the problem and give better returns on the investments made by the institution. A more specific focus on prevention and protection of workers is, therefore, necessary during academic training.

\section{INTRODUCCIÓN}

El Programa Nacional de Inmunizaciones (PNI) se instituyó en 1973 como una forma de coordinar acciones caracterizadas, hasta entonces, por la reducida cobertura. En el área de la salud, es una prioridad nacional, con responsabilidad de los gobiernos federal, estatal y municipal. El alcance de los objetivos y la adopción de estrategias con un mínimo de unidad exigen la articulación de estas instancias, para compatibilizar actividades, necesidades y realidades, en un esfuerzo conjunto ${ }^{1}$.

La Ley no. 6.259, de 30/10/1975, reglamentada por el Decreto oㅜ 78.231, de 12/08/1976, institucionaliza el PNI y define competencias, afirmando que la vacunación es un servicio básico, pasando a planificarse obligatoriamente en el conjunto de las acciones ofrecidas por la red de servicios de salud ${ }^{1}$.

En la Ley Orgánica de la Salud ( $n^{-0} 8.080 / 1990$ ) consta que la salud del trabajador es un conjunto de actividades que se destina, a través de las acciones de vigilancia epidemiológicas y vigilancia sanitaria, a la promoción, protección de su salud, atendiendo a la recuperación y a rehabilitación de la salud de los trabajadores sometidos a los riesgos y quejas derivados de las condiciones de trabajo ${ }^{2}$. La misma Ley objetiva garantizar la revisión periódica de enfermedades originadas en el proceso de trabajo, la prohibición cuando hubiera exposición a riesgo inminente para la vida o salud de los trabajadores, la fiscalización y el control de servicios de salud del trabajador.

Es sabido que en muchas actividades existe mayor riesgo de adquisición y de transmisión de enfermedades infecciosas en el ambiente de trabajo, donde se puede adquirir la enfermedad o ser el vehículo de la misma en su transmisón. Es preciso, por tanto, de acuerdo con la actividad y las características del ambiente de trabajo, definir el grado de riesgo para enfermedades infecciosas tales como la tuberculosis, varicela, rubéola, sarampión, gripe, enfermedades pneumocócicas, enfermedad meningocócica, hepatitis A y B, rabia y poliomielitis ${ }^{3}$. 
La Ley no 4.370/06, de la Prefeitura Municipal de Americana/SP, dispone en su Artículo 1ำ la obligatoriedad de la vacunación de los grupos expuestos a enfermedades inmunoprevinibles, como profesionales y estudiantes del área de salud. Presentando también en su parágrafo único que es competencia de la empresa, entidad o institución a la que estuvieren vinculados, proceder al envío de los profesionales y estudiantes a las Unidades Básicas de Salud, mediante la cumplimentación del Formulario de Solicitud de Vacunas para trabajadores y estudiantes ${ }^{4}$. La misma Ley presenta también en su Artículo 5ํㅜ , que el control de la vacunación de los trabajadores, profesionales y alumnos es responsabilidad de la empresa, entidad o institución a través de los respectivos servicios de medicina del trabajo ${ }^{4}$.

Los estudiantes del Área de la Salud están expuestos a varios tipos de riesgos en sus lugares de prácticas, principalmente al riesgo biológico. Este riesgo está presente directa o indirectamente en su día a día, agrabado por la transmisión y virulencia de los microorganismos presentes en las instituciones de salud ${ }^{11}$.

La Universidad de Brasilia (UNB), en parceria cn el Hospital Universitario de Brasilia (UHB), promovió una campaña para incentivar la prevención de enfermedades y concienciar a profesores, estudiantes y servidores sobre la importancia de la inmunización ofreciendo vacunas contra tétanos, fiebre amarilla, hepatitis $B$, triple viral y rabia humana. Esta campaña atendió no sólo al área de salud, sino también a todos los funcionarios y estudiantes ${ }^{7}$.

Según profesores de aquella universidad, iniciativas como esta son fundamentales para incentivar la vacunación entre adultos, ya que las personas que están en la faja etária de entre 20 a 39 años no manifiestan entender que necesitan ser imunizados, y que sus objetivos se lograron, movilizando y concienciando a la comunidad académica sobre la importancia de la vacunación. El primer día se vacunaron 300 estudiantes, profesores y trabajadores. Cerca de 500 personas se vacunaron en la última ronda de la campaña, dos tercios más que la media de las tres primeras rondas. En la tercera fase, el $60 \%$ de los inmunizados estaban en la faja etária de 18 a 30 años. En el primer semestre, se vacunaron aproximadamente 1,4 mil personas. La alta demanda estuvo motivada por el hecho de que la vacunación se realizó dentro del campus, en lugares de gran circulación ${ }^{7}$.

Basándonos en estos datos, el objetivo de este estudio es conocer la percepción de los estudiantes del curso de graduación en enfermería sobre su necesidad de inmunización en cuanto que trabajadores del área de la salud.

\section{MATERIAL Y MÉTODOS}

El presente estudio se caracterizó por su enfoque cualitativo, de tipo exploratorio ${ }^{9}$, y se dearrolló en la Universidad Federal de Rio Grande, junto al Curso de Graduación en Enfermería, que en 2008 contaba con 210 estudiantes matriculados.

El período de colecta fue marzo/abril de 2008. E instrumento utilizado para la colecta de datos fue un cuestionario semi-estructurado, con preguntas abiertas y cerradas.

Los cuestionarios se distribuyeron a los 35 participantes, estudiantes que demostraron interés en formar parte del estudio, estipulándose un plazo de siete días para su devolución. Se garantizó el anonimato de los mismos, presentándose sus comentarios a lo largo de los capítulos Resultados y Discusión, con la identificación A1, A2, A3,... A35. 
El análisis y la organización de los datos se produjo a través de: pré-análisis, exploración del material, organización de resultados. En la fase de pré-análisis, se realizó la lectura y codificación de los cuestionarios, separando las respuestas por semejanzas y diferencias. En la fase de exploración, hubo lectura exhaustiva y reorganización del material recolectado; finalmente fueron elaborados los contenidos por categorías, asociándose en cinco categorías: la importancia de la inmunización; las actividades de riesgo; acceso a las informaciones sobre inmunización; las vacunas indicadas a los trabajadores de salud; las sugerencias para contextualizar la necesidad de inmunización.

Se siguió la Resolución 196/96 del Consejo Nacional de Salud del Ministerio de Salud ${ }^{10}$. Los participantes fueron informados del objetivo y de la metodología del estudio, solicitándose su Consentimento Libre y Esclarecido. El proyecto de investigación fue aprobado por el Comité de Ética en Investigación en el Área de Salud - CEPAS/FURG, por el parecer nº 20/2008.

\section{RESULTADOS}

Del total de estudiantes del curso (210), participaron de este estudio 35 estudiantes matriculados de primero a cuarto año del curso; 22 son del sexo femenino y 13 del sexo masculino. La edad de los estudiantes varió de 19 a 39 años.

A partir de las informaciones contenidas en los cuestionarios, las respuestas se clasificaron por semejanzas y diferencias. Posteriormente, se agruparon en cinco categorías, previamente definidas en la metodología.

\section{Categoría 1 - La importancia de la inmunización}

A través de sus respuestas, los estudiantes reconocen la importancia y necesidad de la inmunización y del conocimiento de la prevención de varias enfermedades transmisibles, principalmente por tener contacto directo con los materiales orgánicos de varios clientes. De acuerdo con los participantes de este estudio, estar vacunados es de vital importancia, pues

"[...] estamos con pacientes, la mayor parte de las veces, con enfermedades transmisibles. Entonces, estar inmunizado contra [...] enfermedades disminuye el riesgo de adquirirlas, ya que como somos estudiantes y estamos aprendiendo, el riesgo de que ocurra algún accidente es grande, con material taladro-cortante, por ejemplo." (A10).

" [...] vivimos en un ambiente sujeto a contaminaciones, agujas, basura hospitalaria y pacientes contaminados."(A21)

"...incluso cuando los estudiantes entramos en contacto con situaciones de riesgo para nuestra salud y la vacunación es una de las formas de protección del estudiante." (A7).

\section{Categoría 2 - Las actividades de riesgo}

En cuanto a la percepción de riesgos en las actividades, los estudiantes de enfermería se sienten expuestos a los riesgos derivados de la realización de procedimientos invasivos, principalmente debido a las alteraciones de salud de clientes atendidos en su cotidiano de trabajo. 
"Procedimientos invasivos, comunicación con usuarios en grupo de riesgo, ambientes cerrados y poca ventilación, en el cual realizamos actividades, utilización de equipamientos de protección individual ineficaz." (A19).

"Son innumerables las actividades en que necesito de vacunación, principalmente el contacto con los pacientes en el hospital, pues durante toda la vida académica tenemos mucho contacto con enfermedades de alto riesgo de contagio, pero que tienen un método de prevención muy fácil que es la vacunación." (A24).

"... Tener contacto con secreciones de los pacientes, accidentes con pacientes con Hepatitis y HIV y otras, estar expuesto a enfermedades como tuberculosis, cuya forma de transmisión es a través del habla, respiración, secreciones (esputo)." (A25).

Solo un estudiante refirió que la necesidad de vacunación es esencial para la realización de sus actividades cuando está en contacto con pacientes en el hospital. Así, es posible entender que reconocen la necesidad del cuidado en los más diversos campos de actuación tanto curriculares como extracurriculares.

\section{Categoría 3 - Acceso a las informaciones sobre inmunización}

En los entornos referentes a las informaciones y orientaciones sobre las vacunas indicadas para su grupo de edad y desarrollo de actividades, se identificó que entienden que tienen poco conocimiento, a pesar de que cursan una disciplina en la cual está especificamente trabajado el tema "inmunizaciones", mas no puntualmente las vacunas de los trabajadores. Los estudiantes mencionan como fuentes de información referentes a la inmunización:

“[...]internet, en el sitio del Ministerio de Salud." (A27).

"carpeta de la Secretaría Municipal de Salud, Secretaría Estadual de Salud y el Manual de Inmunizaciones del M.S." (A34).

"internet, a través de campañas y colegas [...]." (A33).

"[...] libros e internet." (A15).

"[...] carpeta [...]." (A18).

En los relatos sobre las formas utilizadas para accesar las informaciones, los estudiantes citaron con más frecuencia los recursos "externos", y pocos fueron los que mencionaron las disciplinas de la graduación, lo que nos lleva a preguntarnos sobre los enfoques desarrollados en el curso de graduación y la utilización de material para consulta.

\section{Categoría 4 - Las vacunas indicadas a los trabajadores de la salud.}

Las respuestas abajo ilustran el conocimiento de los estudiantes respecto a las vacunas necesarias a los trabajadores del área de la salud:

“Tétanos, Hepatitis, Rubeola, BCG." (A1).

"Hepatitis." (A2).

"Hepatitis B, anti-tetánica, hizo las dos." (A18). 
"BGC, Hepatitis B, dt, SRC o rubeola, VO." (A25.)

En relación a las respuestas sobre las vacunas ya recibidas:

"No, estoy en proceso con el esquema de la hepatitis B. La anti-tetánica está atrasada."(A11).

"iNo estoy seguro! Me puse la vacuna contra la rubeola el año pasado, obs: no estoy al dia con las vacunas." (A12).

"Realicé todas." (A22).

"No, porque no realicé todas las dosis de la vacuna anti-hepatitis." (A26).

"No estoy seguro" (A31).

\section{Categoría 5 - Las sugerencias para contextualizar la necesidade de inmunización}

Al ser preguntados sobre acciones que consideran importantes para el cambio de enfoque y comprensión del tema, presentaron este orden:

"[...] deben ser orientados también en clase a realizar todas las vacunas necesarias." (A6).

"Gustaría que los profesores nos orientasen más sobre la realización de las vacunas, mayor divulgación en los murales de vacunación de los pasillos, organizar grupos de vacunación a los estudiantes, cuando el alumno visita el centro de salud por primera vez debe presentar la cartilla de vacunación... conferencias de profesionales de la salud a los estudiantes de primero son siempre bienvenidas... esos encuentros nos proporcionan información y despiertan nuestro interés [....]."(A12)

"Encuentro que deberían ser más discutidos [...]." (A15).

"[...] abordando un contenido en el $1^{\circ}$ semestre [...]". (A24).

"Debería tener un calendario vacunal para estudiantes y funcionarios." (A28).

"El medio académico debería estar más informado acerca de la necesidad de vacunación para el auto cuidado." (A34).

A pesar de relatar que saben la importancia y necesidad de la vacunación y cuáles vacunas deben recibir, hay carencia de conocimientos sobre el asunto. En este momento, es interesante retomar la discusión de que tener solo informaciones no es suficiente.

\section{DISCUSIÓN}

En los últimos años, a consecuencia de la propagación de enfermedades transmisibles, como por ejemplo la gripe A y la Fiebre amarilla, se ha observado en Brasil un crecimiento de la aceptación de la vacunación entre los profesionales del área de la Salud, aunque la prevalencia sigue siendo insatisfactoria, especialmente cuando se considera que la mayoría de las vacunas están disponibles gratuitamente en la red pública de salud. 
La inmunización es parte esencial de los programas de control de infeción en salud ocupacional, pues asegura al trabajador del área de salud reducción en los riesgos de adquisición de enfermedades inmunoprevenibles, así como la reducción en el número de individuos y profesionales susceptibles, reduciendo así el riesgo de transmisión de enfermedades de los profesionales a los pacientes y viceversa ${ }^{11}$.

La Sociedad Brasileña de Inmunización (SBIm) recomienda un calendario vacunal ocupacional, reconoce que los profesionales de salud están bajo riesgo significativo de contraer / transmitir enfermedades previnibles por inmunización y preconiza las vacunas contra hepatitis $A$ y $B$, gripe, sarampión, paperas, rubeola, difteria, tos ferina, tétanos y varicela $^{18}$.

En 2004, el Ministerio de Salud instituyó en todo el territorio nacional la inmunización contra la hepatitis $B$ en adolescentes con rango de edad de 11 a 19 años, lo que desde 1986 era solamente para recién nacidos, y ese mismo año hizo la vacunación obligatoria de todas las vacunas a todos los ciudadanos ingresados en escuelas, desde guarderías, universidades a empresas ${ }^{16}$. Entre tanto, corroboramos con algunos autores ${ }^{11}$ que refieren que la realidad revela un gran número de profesionales y estudiantes que no tienen su cartilla de vacunación actualizada, sin mencionar aquellos que desconocen su histórico vacunal. En nuestra investigación, pudimos notar que muchos de los sujetos, con la edad mínima de 19 años, no recibieron la completa inmunización disponible en la red pública contra la hepatitis B y Triple viral (sarampión, paperas y rubeola) y la BCG, insistiendo en la necesidad de que los futuros profesionales busquen la prevención contra estas enfermedades.

Destacamos también que la baja adhesión de los graduandos a la vacuna contra la rubeola es preocupante, pues gran parte de los estudiantes del área de la salud son del sexo femenino, lo que también fue constatado en estudio realizado y con estudiantes de enfermería de Minas Gerais ${ }^{17}$.

Se percibe una "incoherencia" entre lo que dicen ser importante y el "comportamiento" de promoción y prevención. Resulta inquietante que el estudiante no esté seguro de qué vacunas realmente recibió, especialmente por el hecho de que la frecuencia de diagnósticos de Hepatitis $B$ y de Hepatitis $C$ han presentado aumento en los últimos años. Es importante resaltar que la inmunización contra la Hepatitis B y contra el Tétanos, son consideradas como precaución estándar ${ }^{1}$.

El accidente laboral, incluyendo la exposición a material biológico, es tema relevante en el área de salud. Los riesgos de infección de los estudiantes de enfermería son como mínimo iguales a los de los profesionales ya insertos en las unidades de salud. En este caso, el riesgo se vuelve más preocupante, pues estos participan en una serie de actividades que los exponen a sangre, secreciones y/o fluídos corpóreos, mientras están en proceso de desarrollo de habilidades técnicas, convirtiéndose en un agravante que potencia este riesgo ${ }^{19}$.

En un estudio cuyo objetivo fue describir la epidemiología de las exposiciones ocupacionales de alumnos de tercer año de Medicina e identificar los factores de riesgo asociados que puedan ser modificados con la intención de reducir la exposición al riesgo, se encontró que $60(9,3 \%)$ de los estudiantes se expusieron a material biológico y que entre los tipos de accidentes $36(60,0 \%)$ fue exposición íntegra de la piel o membrana mucosa a sangre y fluídos corpóreos y $24(40,0 \%)$ hubo lesión por instrumento taladrocortante ${ }^{20}$. 
Otra investigación, realizada en 2003, verificó que de 7649 accidentes ocurridos en el período de 1995 a 1998, $4587(60,0 \%)$ de los profesionales afectados eran enfermeros y estudiantes de Enfermería, y que $77,6 \%$ de las lesiones fueron provocadas por agujas, con sangre, llamando la atención la exposición ocupacional de esta categoría profesional. En la actuación de los profesionales del área de enfermería el tiempo dispensado al paciente/cliente, la naturaleza de los procedimientos realizados y el uso frecuente de objetos taladrocortantes, hace que los accidentes ocurran con mayor frecuencia, justificando el porqué de un mayor número de accidentes entre los enfermeros ${ }^{19}$.

De entre las actividades identificadas como de riesgo, están aquellas donde hay exposición a los fluídos corporales de la clientela, especialmente la sangre, siendo el uso de material taladrocortante, la que más aparece. Estos datos son coherentes con la frecuencia identificada en la literatura en que $50 \%$ del total de accidentes de trabajo, ocurre por el uso de taladrocortantes ${ }^{12}$.

Estos estudios corroboran nuestros hallazgos y confirman, una vez más, la vulnerabilidad del alumno a los accidentes, y nos lleva a repensar nuestro papel en relación a la prevención y control de la infección en los ámbitos académico y laboral. Demuestran también que a pesar de que las instituciones de enseñanza orientan a sus alumnos sobre la inmunización, esta enseñanza ha sido débil, no explicitando cuáles vacunas deben ser recomendadas a los profesionales del área de salud, incluso estando preconizado por la Comisión de Control de Infección Hospitalaria $(\mathrm{CECIH})^{11}$. Así, se incrementa la necesidad de implantación e implementación de políticas no sólo para los accidentes ocupacionales en las Instituciones de Enseñanza de Salud, sino también para la inmunización de los graduandos, o sea, para todas las medidas necesarias para la prevención y control de las infecciones.

Nuestros resultados fueron semejantes a los de dos estudios brasileños publicados en $2006^{11}$ y $2010^{14}$, evidenciando una orientación compartimentalizada y de enfoque fragmentado, lo que dificulta agregar el conocimiento de forma consistente y aplicable al cotidiano del profesional de salud. Coincidimos con algunos autores ${ }^{11}$ que afirman que a pesar de constituirse la disciplina curricular en la mayor fuente de información, observamos una disolución de este contenido en una serie de disciplinas, desde las del ciclo básico, común a todos los cursos, como Bioquímica, Inmunología y Microbiología, a las disciplinas específicas de cada curso, no habiendo consenso en cuanto a una disciplina específica, siendo citadas aproximadamente 32 disciplinas diferentes.

Estudio semejante al nuestro sobre el papel de las instituciones de enseñanza superior en la prevención de las enfermedades inmunoprevenibles ${ }^{11}$, revela que esta es la forma más eficaz de prevención y protección contra exposición a la contaminación de virus en el ambiente de trabajo. Aparece en su texto que, el Ministerio de Salud de Brasil, en 2004, a través de la Portaria $\mathrm{n}^{0} 597^{16}$, torna obligatorio, en universidades, el comprobante de vacunación actualizado, de acuerdo con el calendario de vacunación y grupo de edad establecidos por el Programa Nacional de Inmunizaciones. Su anulación, determinada por el Ministerio de Salud, se produjo en 2006, bajo la Portaria GM/MS no $1.602^{15} \mathrm{y}$, se refiere principalmente, a la exposición y riesgos laborales inherentes a las actividades profesionales de los graduandos de cursos del área de salud.

Aprobamos la sugerencia de los autores ${ }^{14}$ en relación al control de revocación en la inmunización individual, que se daría por medio del "trote" al estudiante con ocasión del acceso a la universidad, cuando los estudiantes de primero serían evaluados en cuanto a la situación vacunal y enviados para su regularización. $O$ incluso, involucraar en la implementación de programas sistemáticos y periódicos de vacunación a los estudiantes, 
eventualmente en colaboración con la Dirección de las instituciones, que podrían tener el mismo resultado potencial en la detección y regularización de omisiones en la cobertura vacunal $^{14}$.

La Universidad Federal de Rio Grande - FURG, a través del Proyecto T-HE-R Saúde, en el año 2007, realizó actividades para actualizar el calendario vacunal de trabajadores del Hospital Universitario Dr. Miguel Riet Corrêa Jr (HU) de la FURG, alumnos y docentes de los cursos de graduación en Enfermería y Medicina, servidores administrativos, y estudiantes del Curso Técnico de Enfermería (Colégio Técnico Dr. Mario Alquati), ofreciendo vacunas contra Hepatitis B, Tríple Viral (TV) y Difteria y Tétanos (Dt) ${ }^{8}$.

Este proyecto ${ }^{8}$ contó con la participación voluntaria de los estudiantes de enfermería para la administración de las vacunas. A través de las actividades desarrolladas se buscó viabilizar el contacto del estudiante con todas las normas y con la legislación pertinente la regulación de la prevención y control de infección, pues cuanto más precoz sea ese contacto, mayor será la posibilidad del futuro profesional de concienciarse y asimilar las prácticas preconizadas $^{2}$.

Acreditamos que el conocimiento construído en la academia, tiende a ser aplicado y repetido por el trabajador cuando está inserto en el mercado de trabajo. En lo que se refiere a la inmunización, en la graduación, los egresados deben comprender los aspectos teóricos y prácticos del control y prevención de las infecciones relacionadas con los servicios de asistencia a la salud (IRSAS), pues constituirán elementos de una actuación profesional coherente.

Resaltamos también que no existen muchas producciones relacionadas con la inmunización de estudiantes y trabajadores de enfermería. Así, consideramos necesario producir más en esta temática, pues así se podrá reflexionar también la valorización del cuidado y de la orientación de enfermería.

\section{CONSIDERACIONES FINALES}

Este estudio exploró el conocimiento y la percepción de los estudiantes del curso de Graduación en Enfermería de la FURG acerca de su necesidad de inmunización, siendo un espacio para llevar a los participantes a la reflexión sobre la necesidad de su inmunización, como resultado del contacto con situaciones en las que hay riesgos por estar expuestos a agentes patogénos virales y bacterianos, en la mayor parte de los campos de actuación, tanto en actividades curriculares como extracurriculares.

A través de los resultados obtenidos, se puede considerar que los estudiantes de enfermería participantes reconocen las inmunizaciones como um modo de protección, refiriendo fragmentos y necesidades en relación a la misma, manifestando la expectativa de que recibieron las informaciones a lo largo del curso. Al mismo tiempo que, casi por unanimidad, destacan la importancia de la prevención, no todos mantienen el calendario vacunal actualizado, siendo que algunos no están seguros de qué vacunas recibieron.

Este contexto permite reflexionar sobre la complementación en la manera de abordar el tema en la institución, estimulando un enfoque más específico de protección y prevención de los trabajadores durante la formación académica; hacer obligatorio el comprobante de vacunación actualizado para todos los estudiantes que tienen que realizar prácticas, conforme Portaria MEC 597/04. 
De entre las Sugerencias para la continuidad del estudio pueden enumerarse: aumento del $\mathrm{n}^{\circ}$ de participantes; mantenimiento del Proyecto THER Saúde; inclusión del tema en las Aulas Inaugurales del curso; contribución para COMCUR de Enfermería con informaciones sobre riesgos y las vacunas indicadas a los trabajadores del área de la salud; sensibilizar a los estudiantes sobre su necesidad de inmunización.

De este modo, si reflexionamos sobre este asunto y aumentamos nuestro conocimiento sobre los riesgos que pueden encerrar las diferentes situaciones, así como sobre las medidas que deben implementarse para prevenirlos, estimularemos la concienciación de los estudiantes del área de la salud sobre la importancia de su inmunización en el futuro, como trabajadores cuidadores, favoreciendo que tengamos otro tipo de posición, para nosotros mismos y para nuestros clientes.

Esperamos que este trabajo contribuya a la propuesta y mantenimiento por parte de la institución de un programa relacionado con la prevención de los riesgos laborales de los estudiantes, que son los futuros proesionales del área de la salud.

\section{REFERENCIAS}

1. Brasil, Ministério da Saúde. Doenças Relacionadas ao Trabalho, Manual de

Procedimentos para os Serviços de Saúde-Representações no Brasil da OPAS/OMS.

Brasília (DF); 2001.

2. Brasil Lei nº. 8080/90 de 19 de setembro de 1990- Art.6, \&3ํ. Diário Oficial da República Federativa do Brasil, Brasília , 1990.

3. Ballalai I, Migowski E: Imunização e prevenção nas empresas, Um guia de orientação para a saúde dos negócios e do trabalhador. 2006 p.01-24, Rio de Janeiro. Extraído de [http://www.sbim.org.br], [acesso em 12 de outubro de 2007].

4. Hetze EJ. Imunização dos trabalhadores (Prefeitura Municipal da Americana 04/07/2006) Revista Jovem Médico .242- 243 abril/00, publicação oficial: Jornal O Liberal, de 07/07/2006 Extraído de [http://www.americanasp.gov.br/legislacao/leis/4370.htm], [acesso em 25 de agosto de 2007].

5. Machado JMH. Processo de vigilância em saúde do trabalhador. cadernos de saúde pública, artigo, vol.13 Suppl: 2 Rio de Janeiro 1997.

6. Oliveira MG. Aspectos epidemiológicos dos acidentes de trabalho num hospital geral. Ver. Bras. Saúde Ocupacional, 10 (40), 1982.

7. UNB, Universidade de Brasília. Saúde: Vacina também é coisa de gente grande. Textos UNB Agência, 05 de maio de 2006. Extraído de [http://www.secom.unb.br/unbagencia/ag0606-09htm], [acesso em 15 de agosto de 2007].

8. Maas CG, et al. Projeto T-HE-R Saúde: vacinação de profissionais de saúde e estudantes dos cursos de enfermagem e medicina da Furg, Rio Grande, março/2007.

9. Minayo MCS. O desafio do conhecimento. $2^{\underline{a}}$ ed. Pesquisa Qualitativa em Saúde São Paulo-Rio de Janeiro, 2004.

10. Brasil. Resolução 196/96 do Conselho Nacional de Saúde. Diário Oficial da República Federativa do Brasil, Brasília, 1996.

11. Santos SLV, et al. O papel das Instituições de ensino superior na prevenção das doenças imunopreveníveis, Revista Eletrônica, 08 (01): 91 - 98, 2006.

12. Ballalai I. Sociedade Brasileira de Imunizações. Esquema de imunização indicado em serviços de saúde, Abresst- Seminário NR32- Gestão em Segurança e Saúde no Trabalho em Serviços de Saúde, abril/2007 - São Paulo.

13. Queiroz MCB. Biossegurança. In: Oliveira AC. Albuquerque CP, Rocha LCM. Infecções hospitalares-abordagem, prevenção e controle. Rio de Janeiro: MEDSI, 1998, 183-195. 
14. Cheuhen Neto JA et al. Situação vacinal dos discentes da Faculdade de Medicina da UFJF-MG. Rev. bras. educ. med. [online]. 2010, 34(2): 270-277.

15. Brasil. Ministério da Saúde. Portaria №1602, de 17 de Julho de 2006. Institui em todo o território nacional, os calendários de Vacinação da Criança, do Adolescente, do Adulto e do Idoso. [online]. [acesso em 6 abr. 2008]. Disponível em: http://dtr2001.saude.gov.br/sas/PORTARIAS/Port2006/GM/GM-1602.htm

16. Brasil. Ministério da Saúde. Portaria №597, de 8 de Abril de 2004. Institui, em todo território nacional, os calendários de vacinação. [online]. [acesso em 6 abr. 2008]. Disponível em http://dtr2001.saude.gov.br/sas/PORTARIAS/Port2004/GM/GM-597.htm

17. Leite JCA.; Ribeiro JL; Neme NC. Acompanhando a situaçào vacinal de graduandos de Enfermagem: relato de experiencia do projeto de Extensão Fique Esperto Vacine-se já. In: 60 Congresso Brasileiro de Enfermagem, 2008, Belo Horizonte. Espaços de Cuidado, espaço de poder: Enfermagem e cidadania. Brasilia : ABEn-Nacional, 2008.

18. Sociedade Brasileira de Imunizações. Calendários de vacinação. (on line). Disponível em: <http://www.sbim.org.br/programas.htm>. Acesso em: 30 set. 20010.

19. Santos SV. Situação vacinal e exposição a material biológico: o papel das instituições de ensino superior na forma- ção de graduandos na área de saúde no estado de Goiás Goiânia, 2006. 92p : il. Dissertação (Mestrado) - Universidade Federal de Goiás, Faculdade de Enfermagem.

20. Birenbaum D; Wohl A; Duda B; Runyon M; Stearns B; Willett M. Medical student's occupacional exposures to potentially infectious agents. Acad Méd, Florida, 2002. 77(2):185189.

21. Tarantola A, Golliot F, Astagneau P, Fleury L, Brücker G, Bouvet E. Occupational blood and body fluids exposures in health care workers: four-year suverllaince from the northem France network. Am J Infect Control, St. Louis, v.31, n.6, p.357-363, 2003.

ISSN 1695-6141 